\title{
Design of PD-PID Control For Quadrotor At Hover
}

\author{
Sanam Memon, ${ }^{2}$ Dr. Nighat Arbab, ${ }^{3}$ Dr. Zeeshan Ali, \\ ${ }^{4}$ Dr. Bhawani S. Chowdhary, ${ }^{5}$ Dr. Irfana Memon \\ ${ }^{1,2,4}$ Professor, MUET Jamshoro, Pakistan, Assistant Professor \\ , ${ }^{3}$ DUET Karachi, Pakistan \\ ${ }^{5}$ QUEST Nawabshah, Pakistan
}

\begin{abstract}
With an enormous succession in development of tiny sensors using MEMS and NEMS technology, developing tiny vehicles drive in the air become center of attention by new researchers. These vehicles can be utilized in different real time applications. Our research work gives a comprehensive description of such type of well known vehicle (i.e., quadrotor). We have used Newton-Euler method to devise mathematical model for quadrotor. We explained the motion of rotors which is not given in existing work. Our proposed system consists of four controllers including elevation controller, heading controller, location controller, and PID controller. PD parameters are generated using self tuning technique. Experimental analysis was done through simulation using MATLAB tool.
\end{abstract}

Keywords-Quadrotor, Quadcopter, Controller, PID, PD, and self tuning.

\section{INTRODUCTION}

Quadrotor were developed initially with an aim to lift the weight [1-2]. The first copter was developed in 1907 by French brothers named as Jacques and Louis. The first copter known as Gyroplane No. 1 [3], could accommodate only one person and hold the weight up to $1274 \mathrm{lbs}$. only. It flew only 2 feet above the surface of earth \& could retain in air only for one minute but it was highly unstable Quadrotor.

In 1920 another development was made in world of Quadrotors, second copter in 1920 was introduced by Étienne Oehmichen known as Oehmichen No.2 [4], this was first stable Quadrotor which could fly at distance of $360 \mathrm{~m}$ and remained in air for about 7 minutes and 40 seconds. Within the same year another copter was made for the use of US army but this was discarded due to its instability. Roughly after thirty years in 1956 Dr. George de Bothezat and Ivan Jerome introduced a highly stable \& practical copter for the use of US army containing two engines. In past few decades several advancement are introduced in Quadrotor there size is reduced noticeably along with high level of stability [5].

\section{A. Working Concept of Quadrotor}

Quadrotor is an under actuated, dynamic vehicle with four equal size rotors at ends of four equal size rods, it produces four input forces to lift the vehicle with 6 degree of freedom [6]. It is very well designed cross structure vehicle whose cross structure is very delicate \& thin but shows robustness because of mechanical linkage of motors. Propellers are connected with each motor through electronic speed controllers. Each rotor will have to produce the specific amount of Torque $\&$ thrust, which could be achieve by changing the pitch \& rotation of one or more than one rotor $\&$ this will help them to lift the quadrotor as shown in Figure 1 below. 


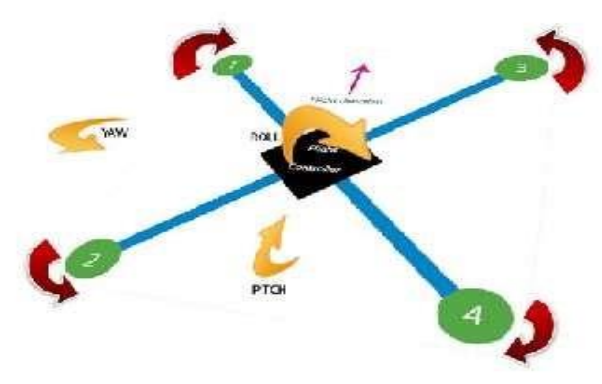

Fig. 1. Rotors on Quadrotor

As it is shown in Figure 1, rotor $1 \& 4$ are performing the rotation in clockwise while $2 \& 3$ are counter clock wise thus in this way they can generate the sufficient Downward Thrust which could lift the quads upward. Unlike helicopters Quadrotors use symmetrical pitched blades for upward motion.

\section{B. Objectives}

Initially the copters were introduced with an aim of lifting the weight \& used by militaries only but after few decades of their development other departments also start to use them for their tasks, such as, firefighting department start to use the copters to take the image of fire captured areas before sending the rescue there, so that one can easily realize the sensitivity of conditions inside the fire captured areas, they are also used commercially to take pictures \& film videos in weddings especially. These are also used whether monitoring department, agriculture department etc.

\section{Advantages of Quadrotor Over Scaled Helicopters}

There are many advantages to Quadrotor over other fixed \& flapping wing rotors:

1- For varying the pitch angle of rotor when copter spins its does not need any mechanical linkage which makes its design simple \& easy maintenance of vehicle [7].

2- Quadrotor uses four rotors which are small in diameter \& occupies small are than helicopters. Due to small size rotors they also require less amount of kinetic energy for take-off $\&$ during flight too which reduces the risk of damages caused when something hit to rotor.

3- It can enter in such areas \& capture the images where difficult for human to enter [8].

4- Due to use of electronic sensors \& control systems in modern copters, made it affordable \& easy to design.

\section{Application of Quadrotor}

Recently researchers have focused on various applications of UAVs [9-30]. Some applications are discussed below-

1. Research Platform: Quadrotor is one of the helpful apparatus for researchers to evaluate \& test multiple concepts in fields like Flight Control, robotics \& Navigation. In running year several international universities used Quadrotors to perform different tasks such as carrying load, capturing images etc.

2. Military \& Law Enforcement: Due to its simple design \& flexible nature it can be used for different purposes, as it can also be used in Military \& law enforcement for Surveillance \& and for transporting the military sorties.

Commercial Use:Quadrotors are widely used for commercial use such as for capturing the images which is not only good job for it because of its autonomous nature but also money saving. It can also 
be used for delivering the goods, for carrying the load \& for capturing the images of weddings $\&$ celebrities.

3. Investigating Purpose: Since Quadrotor is small in size and light in weight, they can get into places, where people cannot get into like caves, holes, tunnels, etc. In 2014, in Tamil Nadu, India Quadrotor was used to investigate the granite scam. Investigators used Quadrotor installed with camera and sensors to get in the tunnel and find the granite in it.

\section{E. Paper Outline}

Our paper is arranged as follows: In Section 2, existing work about the UAVs (Quadrotor) is discussed. In Section 3, modeling with different controller and mathematical formulas for motion of rotors are explained. In Section 4, a detailed description about the proposed methodology is given. The simulation of the model for the PD and PID controller is done and the performance of the system for the controller is studied. Finally Section 5 concludes our work and gives future suggestions.

\section{LITERATURE REVIEW}

This section presents the background survey of the existing literature on optimal control and quadrotor control.

Earlier, the problem of vertical flight was solved by introducing quadrotor. In 1917 after the 14 years of invention of airplane Elmer Sperry introduced an aircraft which was self- stabilizing \& contained barometer, gyroscopes \& servo motor controls as their main components, but it was controlled by human after its successful take off. After this Hewitt-Sperry introduced an automatic airplane which was capable of fly in air $48 \mathrm{kms}$ away from the surface of earth \& drops the bag full of sand when $3.2 \mathrm{~km}$ away from the pre-defined target [31]. The first completely-controlled, unmanned, \& automatic flight flew in 1918 by Curtis-Sperry Flying Bomb, it was proposed by a car \& flew up to the $900 \mathrm{~m}$ distance approximately [32].

Several drones were tested \& developed in period in between First World War \& Second World War, such as larynx was tested in between 1927 - 1929 by Royal Navy, after this in 1931 another drone wad introduced by a British company known as Fairey Queen is was RC-drone (radio-controlled), in 1935 the modified version of "Queen" was introduced known as "DH.82B Queen B", in 1955 the drone named Model 1001 was developed by Beech-craft for the use of US Army. The serious research \& development over UAVs were started after the initiation of $2^{\text {nd }}$ world war when US Army actually concerned about losing their number of pilots in wars \& in hostile territories. In 1946 US Army transformed the B-17 Flying Fortress into the RC-Drone. As initially it was four engine heavy bombers Boeing B-17E introduced in United State by Boeing who took his first successful flight in July 1935. After converting into Drone it was controlled by a transmitter installed into jeep \& on another B-17, during takeoff it was controlled by transmitter on jeep \& during landing by B-17.

After this the drones were began to use in different wars such as cold war, first Gulf Persian war in which they were used by Israelis [32].

From past few decades the increasing need of quad captors especially for military, law enforcement \& commercial use, has led to rise the research for improving the capabilities of Quadrotor. Researchers are improving the Quadrotors day to day by making them advance in fields of multi-craft communication, environment exploration, and maneuverability [33]. Some modern Quadrotor are: Bell Boeing Quad Tilt Rotor, Parrot AR,Drone, Anteos, and Nixie.

\section{MODELING}

\section{A. Kinematics}

Following assumptions have been considered [34]:

1. Un-flexible and uniform arrangement

2. Quadrotor's center of gravity corresponding with origin of static frame of body 
3. Hard and un-flexible propellers

4. Speed of push and strain are relative to the square of propeller's speed

Before discussing the quadrotor's modeling, we start with the discussion on the coordinate frames which will be considered so on. Figure 2 represent the frame of reference of a quadrotor. In this figure, $\mathrm{x}, \mathrm{y}, \mathrm{z}$ variables represent the frame of body, where $\mathrm{N}, \mathrm{E}, \mathrm{D}$ variables represent North, East and downward consequently. It is clearly shown in figure 2, that frame of body locate at the center of quadrotor. The $\mathrm{x}$-axis, $\mathrm{y}$-axis, and $\mathrm{z}$-axis points the $1^{\text {st }}$ propeller, $2^{\text {nd }}$ propeller and $3^{\text {rd }}$ propeller respectively.

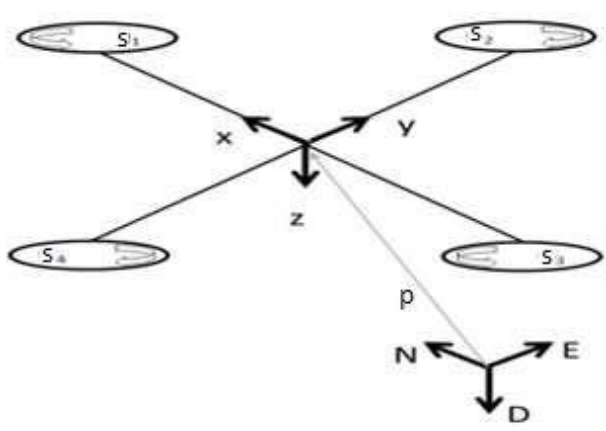

Fig. 2. Reference Frame of Quadrotor

The absolute position of the quadrotor's can be calculated as $\mathrm{P}=[\mathrm{x} \mathrm{y} \mathrm{z}]^{\mathrm{T}}$. Quadrotor's attitude signifies the rotary motion of body frame with the inertial frame. $\mathrm{R}_{M}$ is rotation matrix derived from the product of three basic rotation matrices, represent in Eq. 1.

$\operatorname{Coc} \theta \cos \psi \sin \varphi \sin \theta \cos \psi \cos \varphi \sin \theta \cos \psi+\sin \varphi \sin \psi$

$\mathrm{R}_{\mathrm{M}}=\cos \theta \sin \psi \sin \varphi \sin \theta \sin \psi+\mathrm{c} \theta \mathrm{c} \psi \cos \varphi \sin \theta \sin \psi-\sin \theta \cos \psi$

The angular kinematics is given in Eq. 2.

$$
-\sin \theta \sin \varphi \cos \theta \cos \varphi \cos \theta
$$

$$
\theta=\square \square
$$

Where $\square$ is the transpose matrix representing in Eq. 3 .

$$
\begin{array}{ccc}
1 & 0 & -\sin \square \\
= & \cos \square & \sin \square \mathrm{c} \\
0 & -\mathrm{s} & \square \square \square \\
& \square \square \square & \cos \square \cos
\end{array}
$$

\section{B. Dynamics}

Rotating sub-system (roll, pitch and yaw) and intermediary sub-system (elevation in $\mathrm{x}$ and y location) are known as the two subsystems of the motion of the quadrotor [34].

Newton Euler method is applied to find out the general formula called ad revolving equation of motion.

$$
\square=\square \square+\square * \square \square+\square
$$

Where:

$\square \quad$ = Diagonal inertia Matrix of quad-copter

$=$ Angular rate of body

$=$ Gyroscopic moments due to inertia of rotors

$=$ Moments of quad-copter w.r.t the body frame

The motion of quad-rotor in terms of the rotational equation may be depict as [59]:

$$
\square=\square \square+\square * \square \square+\square *\left[\begin{array}{lll}
0 & 0 & \square \square
\end{array}\right]^{\square}
$$

Where:

Inertia of rotor

$\square$ Relative speed of rotor i.e., $\square=-\mathrm{S} 1+\mathrm{S} 2-\mathrm{S} 3+\mathrm{S} 4$ 
The inertia of rotor is represent through diagonal matrix shown in Eq. (6), where all zero are because of regularity of the quadrotor, and other elements are the ineria's product.

$$
\begin{aligned}
& \text { E } 00 \\
& D_{i}=0 \text { E } 0 \\
& 00 \mathrm{E}
\end{aligned}
$$

Aerodynamic force $\mathrm{F}$ and moment $\mathrm{M}$ produced by the ith rotor of quadrotor [35] is shown in Eq. (7), and Eq. (8) respectively.

Where

$$
\begin{array}{ll}
\rho & =\text { density of air } \\
\text { A } & =\text { area of blade } \\
\square, \square & =\text { coefficients of aerodynamic } \\
r & =\text { radius of }
\end{array}
$$

blade

speed of the rotor $\mathrm{i}$

Eq. (7) and Eq. (8) can rewrite as,

Where $\square$ represent the aerodynamic force constant, $\square$ represent aerodynamic moment constant and $\square$ represent the angular speed of rotor $\mathrm{i}$. The whole moment on $\mathrm{x}$ coordinate is:

$$
=\square \square(-\square \quad+\square)
$$

Moreover, using right-hand-rule on the y coordinate of body frame, propel of rotor 1 produces $+v e$ moment, whereas propel of rotor 3 produces a-ve moment. Therefore whole moment on y coordinate is:

However, on z coordinate moment is generated due to the motion of rotor and it could be calculate as:

Merge above three Eq. (11), Eq. (12) and Eq. (13).

$$
\begin{gathered}
\square \square(-\square+\square) \\
\square=\square \square(\square-\square)
\end{gathered}
$$

The translation formula of motion depends on Newton's $2^{\text {nd }}$ law, which is ermined [34]:

$$
Q d=0+
$$

Here $\square=[\square \square \square]$ distance of Quadrotor from the inertia of frame

Mass of Quadrotor $\mathrm{g}$

acceleration of gravity (i.e., $\mathrm{g}$

$=9.81 \mathrm{~m} / \square$ ) 
Non-gravitational forces applied on the quadrotor in the frame of body. In Eq. (16), $1^{\text {st }}$ and $2^{\text {nd }}$ vector rows are zero values because of null force applied in $\mathrm{x}$ and $\mathrm{y}$ direction. While, $3^{\text {rd }}$ vector row is the summation of the thrust forces produced through four propellers. Moreover, -ve sign shows that the thrust is moving in upward direction.

For obtaining errorless and practical model that should be utilized in simulation, aerodynamic effects should be considered. Practically, aerodynamic effects can be classified as drag forces and drag moments [36]. The drag forces $\square$ can be expressed as:

$$
\square=\square \square
$$

Where $\square$ represents constant matrix known as the coefficient values of aerodynamic, and $\square$ represents the position vector. It implies that the additional force is applied on the quadrotor. Thus, Eq. (15) can be rewrite as:

$$
\square \square=\begin{aligned}
& 0 \\
& 0+\square \square \square-\square
\end{aligned}
$$

Drag moment $\square$ applied on the quadrotor would be expressed as:

In Eq. (19), $\square$ represents constant matrix and $\square$ represents the rate of Euler.

$$
\square=\square \square
$$

Therefore, the revolving equation of motion (i.e., equation 3.5) would now be expressed as:

$$
\square \square \square+\square \times \square \square \times\left[\begin{array}{ll}
0 & 0 \quad \square
\end{array}\right]=\square-\square
$$

Mostly DC motors are used in quadrotor because of large amount of torque and low friction.

Using Kirchhoff's voltage law, the below expression can determine as:

$$
\square=\square \quad \square+\square \quad-+\square \square
$$

In Eq. (21), $\square$ represents motor's resistance and $\square$ represents motor' s inductance of the $\mathrm{i}^{\text {th }}$ motor, represents the current of armature, $\square$ represents voltage and $\square$ is the constant of motor torque which produces emf, e. The inductance of quadrotor could be ignored because it do not depend on large motor. Thus equation 21 can be expressed as:

Going onto the mechanical expression

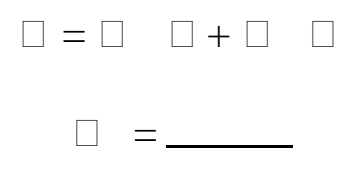

In Eq. 24, $\square$ represents the torque generated by the motor that is equal to $\square . \square$ represents the electric constant of motor. For small motors it is nearly equal to $\square$. $\square$ represents torque produced through propeller system. Replacing the equations of $\square$ and $\square$ gives: 
Now, voltage would be denoted as a function of velocity as follows:

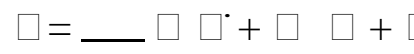

The rotor dynamic can be estimated as $1^{\text {st }}$ order log transfer function with gain and time using MATLAB [14] and was obtained as :

$$
\square(\square \square \square \square \square)=
$$

In equation 27, $\square$ represents actual speed of rotor and $\square$ represents commanded speed of rotor. Brushless DC motor mostly has unique fixed controllers and operates according to PWM signal. RPM of the rotation of the motor directly varies with supplied voltage. This correlation may be identified by a black box detection method for the propeller pair \& motor.

For making commanding issue easier to handle state space model will be formed from obtained mathematical model. X-state vector of the quadrotor is described in Equation (28)

$$
\begin{gathered}
\square=[\square \square \square \square \square \square \square \square \\
\square
\end{gathered}
$$

Eq. (28) can be depicted with degrees of independence shown in Eq. (29),

The $\square$ vector represents the situation of the quadrotor in space. Control-Input-Vector $\square$ comprising of 4-input; $\square 1$ to $\square 4$ be expressed as:

$$
\square=\square \square \square
$$

Where,

$$
\begin{aligned}
& \square 1=\square(\square+\square+\square+\square) \\
& \square 2=\square(-\square+\square) \\
& \square 3=\square(\square-\square) \\
& \square 4=\square(\square-\square+\square-\square)
\end{aligned}
$$

Above equations i.e., Eq. (31) to Eq. (34) will be set in a matrix structure as shown in Eq. (35).

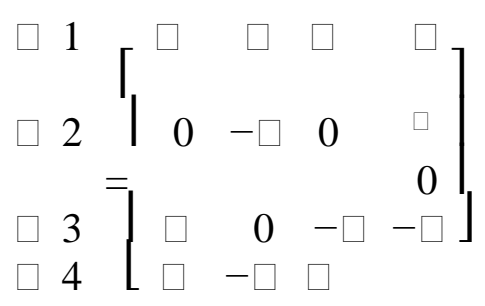

Speed of rotors could be measure through Eq. (36) obtained by reverting Eq. (35). 


$$
\left[\begin{array}{l}
S_{1}^{2} \\
S_{2}^{2} \\
S_{3}^{2} \\
S_{4}^{2}
\end{array}\right]=\left[\begin{array}{cccc}
\frac{1}{4 K_{f}} & 0 & \frac{1}{2 K_{f}} & \frac{1}{4 K_{M}} \\
\frac{1}{4 K_{f}} & -\frac{1}{2 K_{f}} & \frac{0}{0} & -\frac{1}{4 K_{M}} \\
\frac{1}{4 K_{f}} & \frac{0}{0} & -\frac{1}{2 K_{f}} & \frac{1}{4 K_{M}} \\
\frac{1}{4 K_{f}} & \frac{1}{2 K_{f}} & \frac{0}{0} & -\frac{1}{4 K_{M}}
\end{array}\right]\left[\begin{array}{c}
C_{I V} 1 \\
C_{I V} 2 \\
C_{I V} 3 \\
C_{I V} 4
\end{array}\right]
$$

Speed of rotor in given direction can be determined through following Eq. (37) to Eq. (40)

$$
\begin{aligned}
& S_{1}=\sqrt{\frac{1}{4 K_{f}} C_{I V} 1+\frac{1}{2 K_{f}} C_{I V} 3+\frac{1}{4 K_{M}} C_{I V} 4} \\
& S_{2}=\sqrt{\frac{1}{4 K_{f}} C_{I V} 1-\frac{1}{2 K_{f}} C_{I V} 2-\frac{1}{4 K_{M}} C_{I V} 4} \\
& S_{3}=\sqrt{\frac{1}{4 K_{f}} C_{I V} 1-\frac{1}{2 K_{f}} C_{I V} 3+\frac{1}{4 K_{M}} C_{I V} 4} \\
& S_{4}=\sqrt{\frac{1}{4 K_{f}} C_{I V} 1+\frac{1}{2 K_{f}} C_{I V} 2-\frac{1}{4 K_{M}} C_{I V} 4}
\end{aligned}
$$

Moment on quadrotor could be calculate through Eq. (41).

$$
\begin{array}{r}
\square=\square \\
\square \square
\end{array}
$$

Angular accelerations will be calculated using Eq. (42)

$$
\begin{gathered}
\ddot{\emptyset}=\frac{l}{E_{I X}} U_{2}-\frac{R_{I}}{E_{I X}} \dot{\theta} S_{R}+\frac{E_{I Y}}{E_{I X}} \dot{\Psi} \dot{\theta}-\frac{E_{I Z}}{E_{I X}} \dot{\theta} \dot{\Psi} \\
\ddot{\theta}=\frac{l}{E_{I Y}} U_{3}-\frac{R_{I}}{E_{I Y}} \dot{\varnothing} S_{R}+\frac{E_{I Z}}{E_{I Y}} \dot{\varnothing} \dot{\Psi}-\frac{E_{I X}}{E_{I Y}} \dot{\Psi} \dot{\varnothing} \\
\Psi=\frac{l}{E_{I Z}} U_{4}+\frac{E_{I X}}{E_{I Z}} \dot{\theta} \dot{\varnothing}-\frac{E_{I Y}}{E_{I X}} \emptyset \theta
\end{gathered}
$$

\section{RESULTS AND DISCUSSION}

In this section, we will discuss the result of quadrotor model that we have made with four controllers including elevation, head, location, and PID controllers. We will present the result analysis with different required height and varying simulation time. We have performed simulation using a well known tool i.e., MALAB and simulation parameter values were considered same from [37].

\section{A. Proposed Methodology}

Figure 3 represents the methodology used for simulation and analysis. Control System used in this study is Proportional Integral Derivative (PID) Control. Although PD control treats precisely with exceed and buzzing problems linked with relative control. It does not treat the difficulty with the steady-state error. Luckily, it is potential to remove this while using comparatively little gain by accumulation of an integral term to the gain.

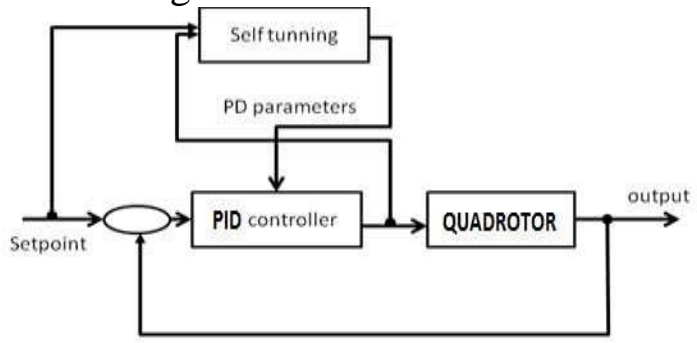

Fig. 3. Proposed Methodology

\section{B. Quadrotor with 4 Controllers}

Figure 4. illustrate the complete designed model with four controllers. 


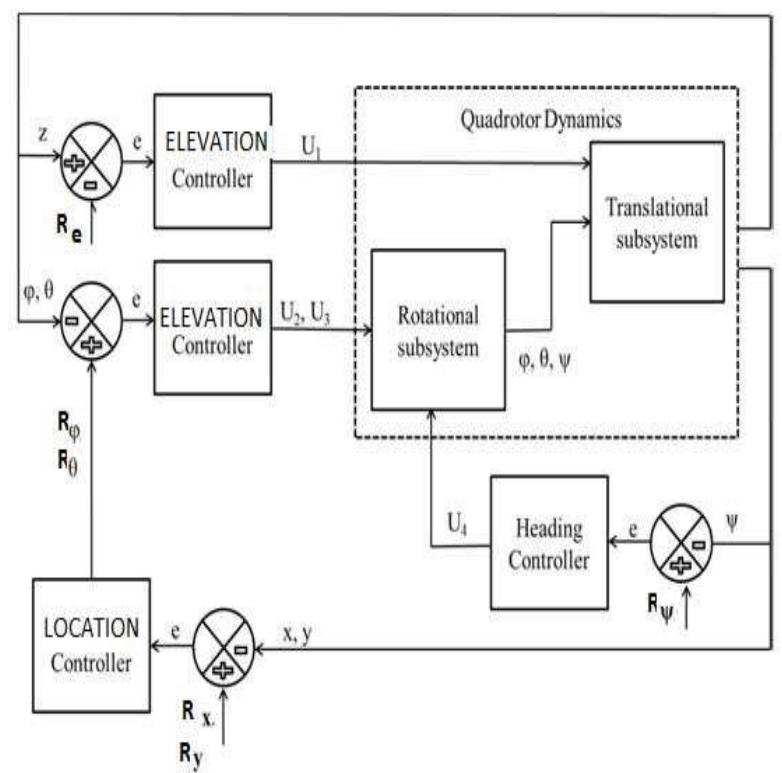

Fig. 4. Block diagram of quadrotor with four controllers

Following equations are used to calculate rate of motion for rotors needed to flow the quadrotor.

In Eq. (44), $\square$ represent the angular velocity of $\mathrm{i}^{\text {th }}$ rotor. So, four forces are applied on quadrotor to bring it in upward direction. Eq. (36) is used to calculate the speed of these four rotors. When $\square$ was support to the open circle activity ( $\square$ through $\square$ ), it was observed that state factors such as: $\mathrm{x}, \mathrm{y}, \mathrm{z}, \theta$, $\Phi, \Psi$ and their derivatives were set aside zero value. The height of quadrotor was altered via increasing speed of rotors. Similarly, move, pitch and yaw are achieved with altering the speed of four rotors. These experiments verify the correctness of the inferred model and the decency of an open circle activity. Also, fundamentals of rotors are integrated with control input computation. After that we extend and integrate an elevation, heading, and location controllers.

\section{Elevation Controller and Heading Controller}

The elevation controller takes e (input signal) that is difference between the $R$ (required elevation) and $\mathrm{z}$ (real elevation), and $U, U, U$ signals will be produced. Whereas, heading controller takes e (input signal) that is difference between the required roll $R_{\phi}$, required pitch $R$ and required yaw $R$ and their real values are $\phi, \theta, \Psi$ respectively. This generate $U$ as output signal.

\section{Location Controller}

The location controller controls the position of quadrotor. It takes location in $\mathrm{x}$ and $\mathrm{y}$ coordinates from transactional subsystem which will be calculate using following equations:

\section{$\square)$}

$\sin \square \emptyset)$

It produces required pitch $\square$ and required yaw $\square$ values. As the quadrotor is working in the region of hover, thus we can select small values for $\square$, and $\square$ respectively. Let we assume $\sin \square \emptyset \equiv \square \emptyset, \sin \square$ $\equiv \square \square \square \square \cos \square \emptyset \equiv \cos \square \equiv 1$ to simplify the above equations,

$$
\begin{aligned}
\square= & \overline{Q_{M}} \quad \text { (sin } \square \emptyset \sin \square+\cos \square \emptyset \cos \square \sin \\
=\frac{-U_{1}}{Q_{M}} & \text { (45) (cos } \square \varnothing \sin \square \sin \square \quad-\cos \square
\end{aligned}
$$


Eq. (47) and Eq. (48) can rewrite in matrix style, and we get:

(51)

$$
\begin{aligned}
& -\sin \square-\cos \square \square \emptyset \\
& = \\
& \text { (49) } \cos \square-\sin \\
& \square \emptyset \quad-\sin \square-\cos \square \\
& = \\
& \cos \square \quad-\sin \square \\
& \square \varnothing \quad-\sin \square-\cos \\
& \cos \square \quad-\sin \square \quad \square= \\
& \square \emptyset \quad-\square \cdot \sin \square-\square \cdot \cos \square
\end{aligned}
$$

$=$$$
\cdot \cos \square-\square \cdot \sin \square
$$

In our simulation, we have set values for $R \varnothing, R$ in the range of $-20^{\circ}$ and $20^{\circ}$ using saturation function for satisfying the above assumption of having small values. However, all input signals to controllers are e to the state of quadrotor and results output signals such as $U$ to $U$ and $R \varnothing, R$.

\section{PID Controller}

In our research work, we have integrated PID controller with quadrotor to control its height. PID controller provides required input signals to quadrotor. Figure 5. illustrate the sketch diagram for a PID controller.

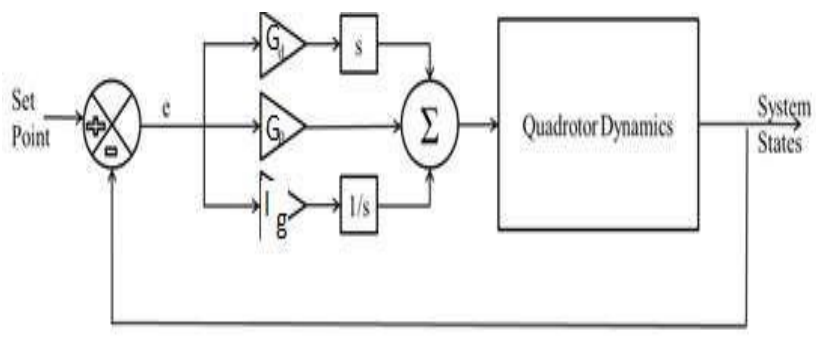

Fig. 5. PID Controller Block Diagram

PID controller generates $\square$ input signals using Eq. (53).

$$
\square=\square(\square-\square)+\square \square-\square+\square \int(\square-\square) \square \square
$$

Where,

$\square$ Proportional gain

Required height

Derivative gain

Rate of change in required height

Integral gain

Furthermore, PID controller generate $\square$ signal to control the roll angle $\Phi$ of quadrotor using Eq. (54)

$$
\square=\square(\square \varnothing-\emptyset)+\square \square \emptyset^{\circ} \square+\square \int(\square \varnothing-\emptyset) \square \square
$$

Where,

$\square \emptyset$ Required angle to roll

$\square \varnothing$ Rate of change in required angle to roll 
Also, PID controller controls the pitch angle $\theta$ of the quadrotor through generating $U$ signal using Eq. (55).

\section{Simulation Result}

$$
\begin{aligned}
& U=\square(\square-\square)+\square \square-\square \cdot \\
& +\square \int(\square-\square) \square \square \\
& +\square \int(\square,-\square) \square \square
\end{aligned}
$$

Where,

Required angle of pitch

Rate of change in required angle of pitch

Moreover, it control yaw using Eq. (56).

Where,

$$
\square=\square(\square-\square)+\square \square-\square
$$

Required angle of yaw

- Rate of change in required angle of yaw

After achieving height control, location of quadrotor is controlled by using Eq.

(57) to Eq. (58) .

$\square=\square(\square-\square)+\square(\square \cdot-\square)+\square \int(\square-\square)$

Where,

Actual location in $\mathrm{x}$ coordinate

Actual location in y coordinate

Required $\mathrm{x}$

location $\square$ Required

y location

Rate of change in required location for $\mathrm{x}$

coordinate $\square$. Rate of change in required

location for y coordinate

We have used an adaptive technique to select gain for PID controller with required height $\square$. Since, we could not observe steady state error with simple PID controller, thus we have converted it with using PD controller via selecting integral gain $\square$ equal to zero. For simulation, PD gain values such as $\square, \square$ were generated with an adaptive tuning technique using following equations:

Where $\square, \square$ are +ve constant values

$$
\begin{aligned}
& \square=\square(\square-\square)+\square \square-\square+\square \int(\square-\square) \square \square \\
& \square(\square)=\square \square(\square) \square(\square)=\square \square(\square) \square(\square)
\end{aligned}
$$

We have analyzed performance by varying required height and simulation time. Firstly, we set required height $\square$ equal to $-2 \mathrm{~m}$ and varies simulation time equal to 2 second. Figure 6 illustrate displacement in $\mathrm{z}$ direction verse time and Figure 7 illustrates velocity in $\mathrm{z}$ direction verse time. 


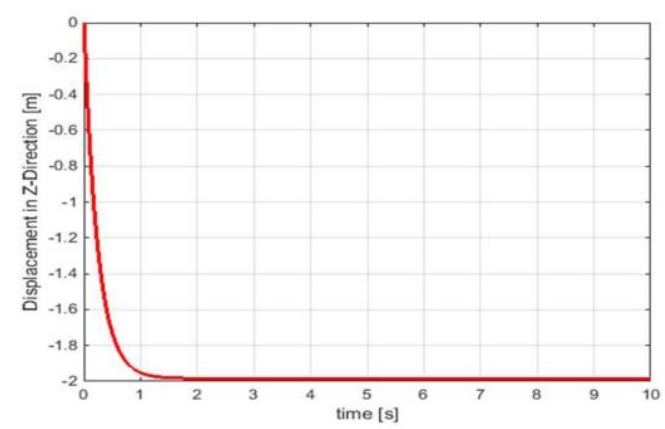

Fig. 6. Displacement VS Time

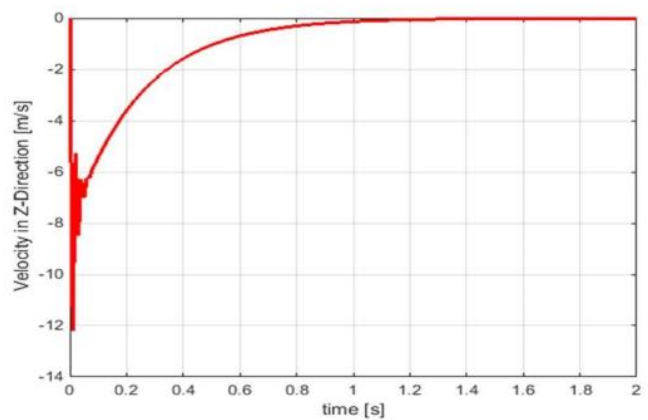

Fig. 7. Velocity VS Time

Figure 8, and Figure 9 shows results with required height $\square$ equal to $-2 \mathrm{~m}$ and varies simulation time equal to 10 second.

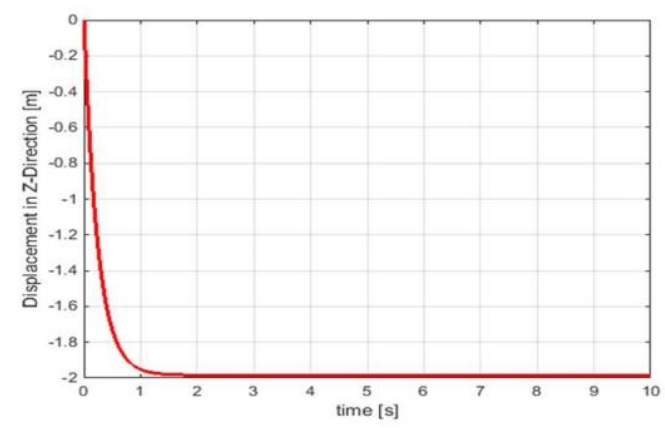

Fig. 8. Displacement vs Time

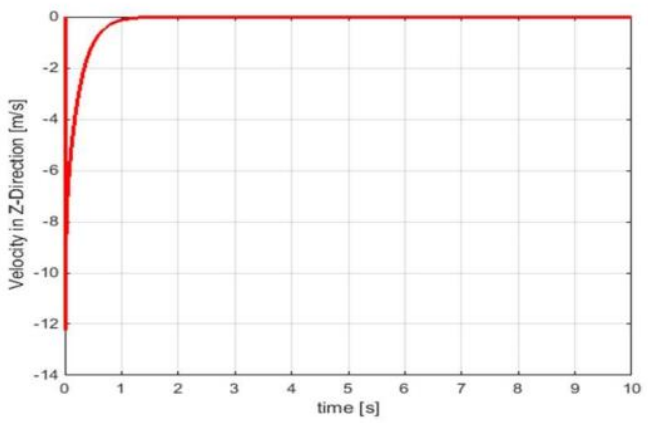

Fig. 9. Velocity VS Time

Then, we set required height $\square$ equal to $-4 \mathrm{~m}$ and varies simulation time equal to 10 second. Figure 10 illustrate displacement in $\mathrm{z}$ direction verse time and Figure 11 illustrate velocity in $\mathrm{z}$ direction verse time. 


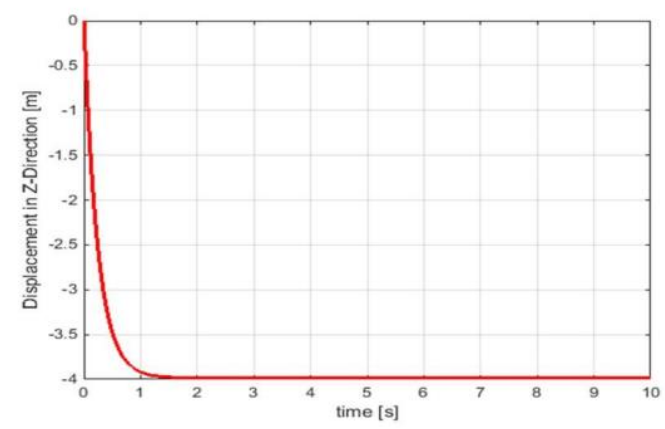

Fig. 10. Displacement VS Time

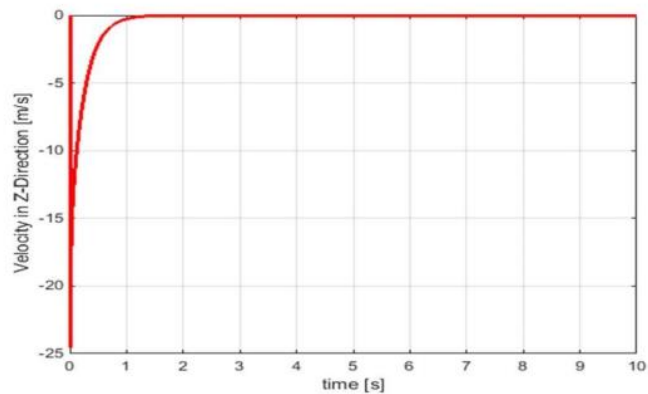

Fig. 11. Velocity VS Time

Finally, Figure 12 shows result for PID Response with variable required height $\square$ varies simulation time equal to 25 second.

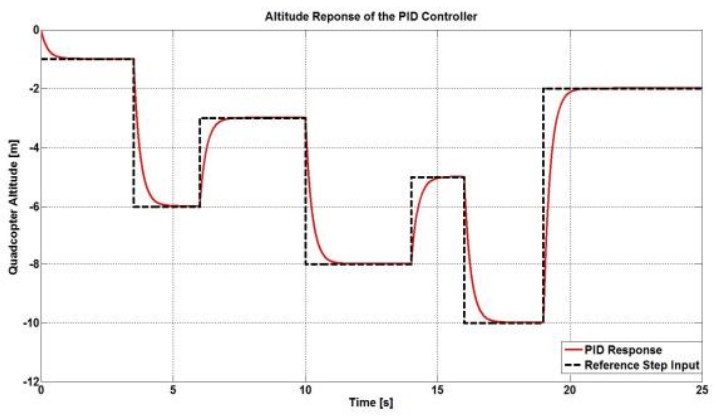

Fig. 12. PID Response

\section{CONCLUSIONS ANS FUTURE WORK}

\section{A. Conclusion}

The aim of our research was to develop a mathematical/precise way for the quadrotor. We build up a controller based method which can be used to alleviate situation/condition of quadrotor such as height, heading, location. We have validated the performance of proposed controllers through simulations. In the existing work, we have found that most of the work has not considered the all external effects applied on quadrotor, such as dynamics of rotors. Therefore, we designed model with four controllers including height controller, heading controller, location controller, and PID controller. It was combined with PD parameters through self tuning technique. The performance analysis was done using well know tool i.e., MATLAB. Simulation was done to assess the performance of used controllers and evaluate their performance using different inputs. Self tuning technique was used to tune the parameters and constant values. Moreover, dynamic responses of the proposed system like resolving time was done using self tuning method. All four controllers operated on the edge operations of quadrotor in the limit of $0 \sim 20$ 。 
of height and heading. Simulation results shows that the used method with PD controller and self tunning gives better performance.

\section{B. Future Work}

In future, we will extend our work and analyze the PD controller in other applications such load fall application and supposing actuator breakdown environment. Then, we will compare it with other controllers. Moreover, it can be analyze with using derivative in the replacement of used required height. In this way, it may be improve the performance of quadrotor. Another situation may be the robustness of proposed technique in the presence of wind which is the very common and possible problem for quadrotor. Moreover, in some of real application, consideration of accurate value for parameters may be not acceptable. Therefore, it will be better to design a method to check the uncertainties of the system. This could be done by combining with integral values. It may be give a countable improvement in real time applications. Last but not least, we will do hardware based analysis of our proposed technique in future work.

\section{REFERENCES}

[1]. Escamilla, R., Diseño, construcción, "instrumentación y control de un vehículo aéreo no tripulado. Tesis de Ingeniería, Escuela Superior de Mecánica y Eléctrica”, Instituto Politécnico Nacional. México D.F, 2010.

[2]. Leishman, J.G. Principles of Helicopter Aerosynamics. New Yorl, NY: Cambridge University Press (2000).

[3]. Bouabdallah, S., P. Murrieri, R. Siegwart, "Towards autonomous indoor micro VTOL”, Autonomous robots, 18(2), 171$183,2005$.

[4]. http://www.dji.com/product/phantom-2-vision-plus

[5]. Chun Fui Liew, Danielle DeLatte, Naoya Takeishi, Takehisa Yairi, "Recent Developments in Aerial Robotics: A Survey and Prototypes Overview", arXiv:1711.10085v2 [cs.RO] 30, Nov 2017.

[6]. http://www.droneybee.com/how-quadcopters-work/

[7]. Pounds, P.; Mahony, R., Corke, P., "Modelling and Control of a Quad-Rotor Robot". In Proceeding of the Australasian Conference on Robotics and Automation, Auckland, New Zealand, December 2006.

[8]. Hoffman, G;;Haung, H.; Waslander, S.L.; Tomlin, C.J. "In the Conference of the Anerican Institute of Aeronautics and Astronautics". Head Hilton, South California, 20-23 August 2007.

[9]. I. Dryanovski, R. G. Valenti, and J. Xiao, “An open-source navigation system for micro aerial vehicles," Autonomous Robots, vol. 34, no. 3, pp. 177-188, April 2013.

[10]. K. Schmid, T. Tomi' c, F. Ruess, H. Hirschmüller, and M. Suppa, "Stereo vision based indoor/outdoor navigation for flying robots," in IEEE/RSJ International Conference on Intelligent Robots and Systems (IROS), pp. 3955-3962, November 2013.

[11]. J. Engel, J. Sturm, and D. Cremers, "Camera-based navigation of a low-cost quadrocopter," in Proc. IEEE/RSJ International Conference on Intelligent Robots and Systems (IROS), pp. 2815-2821, October 2012,

[12]. M. Warren, P. Corke, and B. Upcroft, "Long-range stereo visual odometry for extended altitude flight of unmanned aerial vehicles," The International Journal of Robotics Research (IJRR), vol. 35, no. 4, pp. 381-403, April 2016.

[13]. M. Bloesch, S. Omari, M. Hutter, and R. Siegwart, "Robust visual inertial odometry using a direct EKF-based approach," in Proc. IEEE/RSJ International Conference on Intelligent Robots and Systems (IROS), pp. 2815-2821, October 2012.

[14]. H. Lim, S. N. Sinha, M. F. Cohen, M. Uyttendaele, and H. J. Kim, "Real-time monocular image-based 6-DoF localization," The International Journal of Robotics Research (IJRR), vol. 34, no. 4-5, pp. 476-492, 2015.

[15]. A. Wendel, A. Irschara, and H. Bischof, "Natural landmark-based monocular localization for MAVs," in IEEE International Conference on Robotics and Automation (ICRA), pp. 5792-5799, May 2011.

[16]. Z. Fang and S. Scherer, "Real-time onboard 6DoF localization of an indoor MAV in degraded visual environments using a RGB-D camera," in IEEE International Conference on Robotics and Automation (ICRA), pp. 5253-5259, May 2015.

[17]. R. Konomura and K. Hori, "Visual 3D self localization with 8 gram circuit board for very compact and fully autonomous unmanned aerial vehicles," in Proc. IEEE International Conference on Robotics and Automation (ICRA), pp. 5215-5220, May 2014.

[18]. D. T. Cole, P. Thompson, A. H. Gökto` gan, and S. Sukkarieh, "System development and demonstration of a cooperative UAV team for mapping and tracking," The International Journal of Robotics Research (IJRR), vol. 29, no. 11, pp. 13711399, September 2010. 


\section{Gyancity Journal of Electronics and Computer Science \\ Vol.3, No.1, pp. 24-38, March 2018 \\ ISSN:2446-2918 DOI: 10.21058/gjecs.2018.31003}

[19]. D. Holz and S. Behnke, "Registration of non-uniform density 3D laser scans for mapping with micro aerial vehicles," Robotics and Autonomous Systems, vol. 74, pp. 318-330, December 2015.

[20]. M. Burri, H. Oleynikova, M. W. Achtelik, and R. Siegwart, "Real-time visual-inertial mapping, re-localization and planning onboard MAVs in unknown environments," in IEEE/RSJ International Conference on Intelligent Robots and Systems (IROS), pp. 1872-1878, September 2015.

[21]. G. Loianno, J. Thomas, and V. Kumar, "Cooperative localization and mapping of MAVs using RGB-D sensors," in IEEE International Conference on Robotics and Automation (ICRA), pp. 4021-4028, May 2015.

[22]. A. Bry, C. Richter, A. Bachrach, and N. Roy, "Aggressive flight of fixed-wing and quadrotor aircraft in dense indoor environments," The International Journal of Robotics research (IJRR), vol. 34, no. 7, pp.969-1002, June 2015.

[23]. S. phane Ross, N. Melik-Barkhudarov, K. S. Shankar, A. Wendel, D. Dey, J. A. Bagnell, and M. Hebert, "Learning monocular reactive UAV control in cluttered natural environments," in Proc. IEEE International Conference on Robotics and Automation (ICRA), pp. 1765- 1772, May 2013.

[24]. S. Roelofsen, D. Gillet, and A. Martinoli, "Reciprocal collision avoidance for quadrotors using on-board visual detection," in Proc. IEEE/RSJ International Conference on Intelligent Robots and Systems (IROS), pp. 4810-4817, September 2015.

[25]. J. Müller and G. S. Sukhatme, "Risk-aware trajectory generation with application to safe quadrotor landing," in Proc. IEEE/RSJ International Conference on Intelligent Robots and Systems (IROS), pp. 3642-3648, September 2014.

[26]. E. Graether and F. Mueller, "Joggobot: A flying robot as jogging companion," in Proc. CHI Extended Abstracts on Human Factors in Computing Systems, pp. 1063-1066, May 2012.

[27]. F. Mueller and M. Muirhead, "Jogging with a quadcopter," in Proc. ACM Conference on Human Factors in Computing Systems (CHI), pp. 2023-2032, April 2015.

[28]. M. Cooney, F. Zanlungo, S. Nishio, and H. Ishiguro, "Designing a flying humanoid robot (FHR): Effects of flight on interactive communication," in Proc. IEEE International Symposium on Robot and Human Interactive Communication (RO-MAN), pp. 364-371, September 2012.

[29]. K. Nitta, K. Higuchi, and J. Rekimoto, "HoverBall: Augmented sports with a flying ball," in Proc. Augmented Human International Conference, pp. 13:1-13:4, March 2014.

[30]. J. Pestana, J. L. Sanchez-Lopez, P. Campoy, and S. Saripalli, "Vision based GPS-denied object tracking and following for unmanned aerial vehicles," in Proc. IEEE International Symposium on Safety, Security, and Rescue Robotics (SSRR), pp. $1-6$, October 2013.

[31].https://en.wikipedia.org/wiki/Hewitt-Sperry Automatic Airplane

[32]. Gyrodyne Helicopter Historical Foundation, Gyrodyne Helicopter Historical Foundation, Available: www.gyrodynehelicopters.com/, 2007.

[33]. J. Leishman, Principles of Helicopter Aerodynamics, 2nd Ed., Cambridge University Press, Cambridge, United Kingdom, 2006.

[34]. Amr Nagaty, Sajad Saeedi, Carl Thibault, Mae Seto, and Howard Li. Control and navigation framework for quadrotor helicopters. Journal of Intelligent and Robotic Systems, 70(1-4):1-12, 2013. ISSN 0921-0296. doi: 10.1007/s10846-0129789-z. URL http://dx.doi.org/10.1007/s10846-012-9789-z.

[35]. A. Azzam and Xinhua Wang. Quad rotor arial robot dynamic modeling and configuration stabilization. In Informatics in Control, Automation and Robotics (CAR), 2nd International Asia Conference on, volume 1, pages 438-444, 2010. doi: 10.1109/CAR.2010.5456804, 2010.

[36]. Gergely Regula. Formation control of autonomous aerial vehicles. Phd thesis, Budapest University of Technology and Economics, 2013.

[37]. Samir Bouabdallah, "Design and control of quadrotors with application to autonomous flying", Phd. thesis, Ecole Polytechnique Federale de Lausanne, 2007. 